\title{
Mapeo de Interoperabilidad entre BIM y BPS Software (Simulación Energética) para Chile
}

\author{
Mapping the Interoperability between BIM and Energy Simulation Software for Chile
}

\author{
Danny Lobos \\ Pontificia Universidad Católica de Chile, Chile \\ dlobosc@uc.cl \\ Gerth Wandersleben \\ Universidad del Bio Bio, Chile \\ gerthwwm@gmail.com
}

\author{
Lorena Silva Castillo \\ Pontificia Universidad Católica de Chile, Chile \\ Idsilva1@uc.cl
}

\section{Introducción}

En la actualidad existe una necesidad de crear modelos energéticos de simulación digitales para la verificación del performance de nuestras edificaciones (Attia et all, 2009) (Cho and Chen, 2011), tanto para lograr un buen balance entre la eficiencia energética y la factibilidad económica, como para cumplir con las distintas normas y certificaciones, que rigen el proyecto. Las metodologías de Simulación del Performance del Edificio (BPS, por sus siglas en inglés) han avanzado con éxito debido a la exactitud de los cálculos, las mejoras la interfaz, el uso de amplias bases de datos de materiales, de clima, etc. (Kummar, 2008) Por otro lado los programas BIM (Building Information Modeling) se han masificado en oficinas de Arquitectura, Ingeniería y Construcción. Por su parte los software BIM son conocidos por la poderosa modelación de objetos tales como muros, losas, puertas, ventanas, estructuras, equipos HVAC, etc. de los cuales se hace coordinación interdisciplinarias, tablas de cantidades, planos $2 \mathrm{~d}$, etc. (Eastman, 2008). Sin embargo para el contexto de esta investigación se destacará la capacidad de asignarles información extra a esos objetos, la precisión de la geometría, la posibilidad de crear áreas y zonas del proyecto, etc. Por último existen en Chile las prestaciones que se le exigen a los edificios y que, dependiendo del uso y situación geográfica, determina qué cálculos pueden/deben hacerse usando software de simulación. La mayoría de los especialistas (consultores) en eficiencia energética en Chile, realizan sus modelos energéticos directamente en el software de simulación. Se estima que casi un $30 \%$ del tiempo de la consultoría es destinado al modelamiento del proyecto. Se espera con los resultados, estimular a los Consultores de Eficiencia Energética a exigir modelos BIM a los arquitectos e informar previamente qué ocurre en el proceso de intercambio de información.

\section{Diagnósticos por Área}

\section{Estado del software BIM (Building Information Modeling)}

La mayoría de los modelos hechos en BIM son para fines no asociados a la eficiencia energética, dado que BIM todavía solo es concebido como una herramienta que ayudara en la coordinación, visualización y planimetría de la construcción de edificios. El aspecto clave que posibilita el intercambio es la existencia de los comandos de ZONAS o AREAS en los software BIM. Estos permiten, una vez hechos los muros de un recinto, insertar un volumen virtual contenido en dichos muros. Esto normalmente se usa para computar superficies para conteos de área proyectada, sin embargo, como demostraremos en esta investigación, tiene el potencial de transformarse en ZONAS útiles para el cálculo en el software BPS, ahorrando al consultor la tarea redundante de modelar todo el edificio de nuevo.

\section{Estado del software BPS (Building Performance Simulation)}

Dentro de los software BPS, los formatos más usados para el intercambio con BIM son el DXF, el gbXML y el ifcXML, los cuales son brevemente descritos en la Error! Reference source not 
found.. Un caso especial de estos formatos de intercambio BIMBPS lo constituye el formato RVT (de Autodesk Revit) que será explicado más adelante.

Tabla 1: Formatos de intercambio BIM-BPS. Fuente: Elaboración propia basada en manuales Autodesk y tests. Fuente: Elaboración Propia, 2013

\begin{tabular}{|l|l|l|}
\hline DXF & gbXML & ifcXML \\
\hline $\begin{array}{l}\text { Drawing } \\
\text { Format }\end{array}$ & $\begin{array}{l}\text { Green Building } \\
\text { extensible markup } \\
\text { language }\end{array}$ & Industry Foundation \\
Classes \\
\hline - Geometry & - Building Type & - Full geometric \\
(2d/3d) & - Building Location & description in 3D \\
- Layers & - Geometry & - Object location and \\
Materials* & - Orientation & relationships \\
& - Area & - Properties (or \\
& - Volume & parameters) of each \\
& - Openings Location \& & object. \\
& Size (windows/doors) & - Structural, mechanical \\
& - Lighting, Electrical \& - & and energy analysis \\
& Occupancy Loads* & applications. \\
& - Space Type* & - IfcSpaces \\
& - Condition Type* & \\
& - HVAC Heating \& & \\
Cooling Setpoints* & \\
- Outside Air* & - Materials** \\
** traspasan cuando son agregados a los modelos Revit MEP. \\
reconoce Materiales, pero Revit Architecture no los exporta \\
sorrectamente)
\end{tabular}

Dentro de los casos internacionales desde los ámbitos de la investigación y de la industria que fueron revisados se ha encontrándose especial interés en estos formatos de interoperabilidad, de los cuales hemos podido establecer dos tipos: aquellos formatos basados en Geometría Completa que exportan la geometría del edificio (ejemplo DXF) para cálculos de iluminación y luz día, sombras, viento/ventilación, ganancia solar exterior; y aquellos basados en Espacios (ejemplo gbXML, ifcXML) que exportan información sobre áreas y recintos (generalmente llamados Zonas) para cálculos de desempeño térmico, transmisión de radiación solar, demanda de energía, índices sustentables, etc (Figura 2). La Error! Reference source not found. resume la capacidad de los software BIM de exportar hacia estos formatos.

Tabla 2: Capacidad de los software BIM de exportar a BPS. Fuente: Elaboración Propia, 2013

\begin{tabular}{|l|l|l|l|}
\hline BIM & DXF & GBxml & IFCxml \\
\hline Archicad & DXF & $\mathrm{x}$ & $\begin{array}{l}\text { IFC 2x3 XML } \\
\text { IFC 2x3 XML }\end{array}$ \\
\hline Revit & DXF & GBxml & $\mathrm{x}$ \\
\hline Microstation & DXF & $\mathrm{x}$ & $\mathrm{x}$ \\
\hline Digital Project & & $\mathrm{x}$ & $\mathrm{x}$ \\
\hline Allplan & DXF & $\mathrm{x}$ & $\mathrm{IFCxml}$ \\
\hline
\end{tabular}

Figura 2: Ejemplos de exportación Revit a gbXML. Fuente: Universidad de la Salle. Facultad de Arquitectura.

\section{Estado del Arte en Investigación}

El estado del arte en este tema nos muestra: metodologías de exportación semiautomática, entre BIM y BPS a través de IFC a Energy PLUS, considerando modificaciones de regla GST para la exportación (Bazjanac, 2005-2008). Desarrollo de la interoperabilidad entre las distintas aplicaciones con énfasis en diversos modelos de edificios, incluyendo formatos IFC y $\mathrm{CIS} / 2$ 
(Eastman, 2011). Métodos para estandarizar el contenido de los modelos arquitectónicos en $3 \mathrm{~d}$ de los edificios para la obtención de un modelo interoperable efectiva para permitir simulaciones de eficiencia energética más rápida y fiable durante el ciclo de vida de un edificio (Osello, 2011). Procedimientos y flujos de trabajo, entre Revit, gbXML y Trace 700 (Ali, 2010), permitiendo un traspaso más completo para equipos HVAC. Howell, propone el XML, como protocolo para la trasferencia de subconjuntos o "paquetes" de información relevantes del proyecto como una oportunidad clave para lograr la interoperabilidad entre diferentes disciplinas, como el caso de gbXML. Lobos (2011), discute una nueva aplicación paramétrica basada en BIM para etapas tempranas del diseño, donde la normativa urbana de los edificios determina la forma de éste. La aplicación permite vínculos directos para el análisis de eficiencia energética. Granero (2013) ha introducido en los Currículos de Arquitectura metodologías de comprensión de la Eficiencia Energética mediante la combinación de herramientas BIM-BPS.

\section{Conclusiones}

Los desarrollos de BIM y BPS han alcanzado un alto nivel para sus áreas específicas, sin embargo la interoperabilidad entre ellos es un campo naciente. Existen en el mundo iniciativas aisladas pero exitosas de uso de modelos BIM para simulaciones BPS a través de investigaciones de interoperabilidad. Siendo la interoperabilidad entre BIM y BPS un tema altamente investigado en los últimos 10 años en el hemisferio norte, ha sido enfocado principalmente a prestaciones predeterminadas, usando un software determinado. A nivel de gobierno hay suficiente apoyo a través de normativas. Uno de los formatos con amplias posibilidades de desarrollo, dada su estructura abierta es IFC y el ifcXML.

\section{Objetivos e Hipótesis}

Nuestra hipótesis es que al mapear diversas prestaciones exigidas a los edificios en Chile con la interoperabilidad entre BIM y programas de Simulación Energética (BPS), generaremos un conocimiento que permitirá ahorrar tiempo, aumentar la precisión de los modelos energéticos y creación de un workflow basado en retroalimentación constante entre BIM y BPS.

En el caso de estudio se considera los programas BIM de Arquitectura (AllPlan, Revit, Archicad, etc), los programas de simulación energética (Design Builder, Ecotect, Energy Plus, TAS, etc.). Dentro de los aspectos normativos se consideraron aquellos que tienen que ver con exigencias de Demandas de Calefacción/Enfriamiento, Iluminación, Ventilación, Agua, Acústica, etc.

Se usaron además técnicas de grafos y mapeo de procesos para describir las secuencias de interoperabilidad en cada caso.

\section{Experimentos y Mapeos}

Software de grafos ha sido usado para mapear los software BIM con los BPS y las Prestaciones de Desempeño pudiendo además incorporar en estas conexiones las propiedades de las mismas. Durante el desarrollo de esta investigación se han testeado seis líneas de intercambio de información. Las cuales se describen a continuación y se resumen en la Error! Reference source not found..

Tabla 3: líneas de intercambio de información. Fuente: Elaboración Propia, 2013.

\begin{tabular}{|l|l|l|l|}
\hline formato & caso & BIM -> BPS & Prestación \\
\hline DXF & 1 & $\begin{array}{l}\text { Revit a } \\
\text { Ecotect }\end{array}$ & Iluminación \\
\hline gbXML & 2 & $\begin{array}{l}\text { Revit a } \\
\text { Ecotect }\end{array}$ & $\begin{array}{l}\text { Demanda } \\
\text { Calefacción/Enfriamiento }\end{array}$ \\
\cline { 2 - 4 } & 3 & $\begin{array}{l}\text { Revit a Design } \\
\text { Builder }\end{array}$ & $\begin{array}{l}\text { Demanda } \\
\text { Calefacción/Enfriamiento } \\
\text { lluminación }\end{array}$ \\
\cline { 2 - 4 } & 4 & Revit a TAS & * \\
\hline RVT & 5 & Revit a Vasari & Ganancias Solares \\
\cline { 2 - 4 } & 6 & $\begin{array}{l}\text { Sketchup a } \\
\text { Vasari }\end{array}$ & Ganancia Solares \\
\hline \multirow{3}{*}{$\begin{array}{l}\text { * en estos casos solo se comprobó que el traspaso es correcto pero no } \\
\text { se hicieron cálculos de prestaciones }\end{array}$} \\
\hline
\end{tabular}

\section{DXF: Revit a Ecotect}

Desde el software Revit se exporta la geometría del edificio mediante un archivo de extensión DXF. Luego en Ecotect se importa y se deben considerar "tips" como activar las funciones "Auto Merge Triangles" y "Zone By Item Name". Hay que tener en cuenta que la información importada corresponde a la de geometría del edificio, por lo cual este tipo de intercambio de información está más bien recomendado para el uso en análisis de iluminación y diagramas solares. Los detalles del traspaso se muestran en la Error! Reference source not found.. Este tipo de trabajo tiene las siguientes ventajas: permite analizar geometría DXF desde cualquier software BIM a Ecotect, ei mporta un 95\% del original. $\mathrm{Y}$ encontraremos las siguientes desventajas: geometría compleja traída desde BIM pierde resolución y aumenta el peso del archivo, el modelo generado, no sirve para cálculos de análisis térmico y/o acústico, y por último cambios en el BIM original no se reconocen automáticamente.

\section{gbxml: Revit a Ecotect}

Desde el software Revit se exporta la información mediante el formato gbxml. En este proceso se debe decidir si queremos un traspaso simple (volúmenes $3 \mathrm{~d}$ macizos) o complejo (con aberturas de puertas y ventanas más datos del edificio). Durante el proceso de importación en Ecotect el archivo es reconocido como "Model/Analysis Data". A continuación se debe proceder a modelar los elementos que este tipo de configuración no permite traspasar, tales como elementos de proyectantes de sombra y techumbres, y crear una librería apropiada mediante a la cual se 
podrá asignar el material que le corresponde a cada elemento. Para finalmente poder realizar los estudios de prestaciones de tipos acústicas y térmicas. Los detalles del traspaso se muestran en la Error! Reference source not found.. Este tipo de interoperabilidad tiene las siguientes ventajas: permite usar entre un $80 \%$ y $90 \%$ de la geometría de reciento creada en software BIM y permite agregar información específica para análisis térmico. $Y$ las siguientes desventajas: algunos elementos cambian durante el traspaso, errores de traducción geométrica puede alterar el traspaso, no se pasa correctamente la geometría de los elementos proyectantes de sombra, asignación de la materiales de forma manual induce a error humano, problemas en la identificación de elementos de las categorías puertas y ventanas, en importaciones realizadas desde Autodesk Revit 2013 o superior, Ecotect no reconoce algunos tipos de elemento tomando asignándoles por defecto la clasificación Guess (desconocido), esto debe ser corregido manualmente. Al no existir una actualización del software Ecotect (Autodesk lo compró en 2009 y fue actualizado sólo hasta 2011) se origina error de compatibilidad con cambios en la codificación por lo cual al utilizar Autodesk Revit 2013 o superior, la solución es que el archivo gbXML obtenido debe ser abierto mediante algún editor simple (ejemplo: block de notas, wordpad) y posteriormente guardado cambiando el tipo de codificación del archivo a UTF-8.

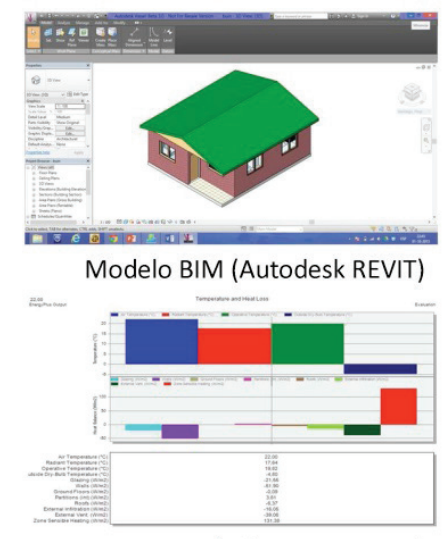

CALEFACCIÓN (DESIGN BUILDER)
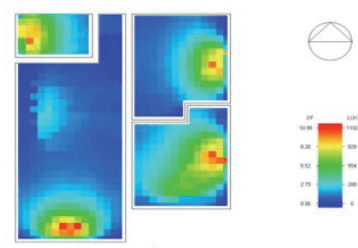

ILUMINACIÓN (DESIGN BUILDER)

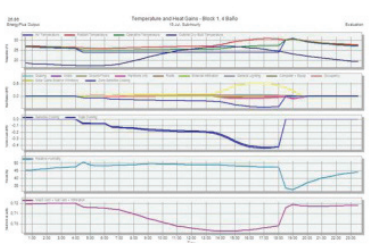

REFRIGERACIÓN (DESIGN BUILDER)
Figura 3: Interoperabilidad BIM-BPS, caso de Revit y Design Builder. Fuente: Elaboración propia, 2013.

\section{gbXML: Revit a Design Builder}

La primera opción para trabajar información elaborada en Revit en Design Builder, es a través del formato gbxml (Figura 3). Para lo cual se realiza un proceso complejo de exportación que incluye: llenar los datos en propiedades del proyecto, editar la configuración de energía, exportación de habitaciones, elegir complejidad de la exportación (simple o con elementos de sombra), cálculo de área y volumen. Una vez importado a Design Builder es necesario incorporar al modelo elementos de sombra y techumbres. Teniendo las correcciones de la volumetría, el siguiente paso será crear una librería de materiales para el proyecto y asignar a cada uno de los elementos el material correspondiente y finalmente agregar toda la configuración correspondiente a locación, función del edificio y otros que sirven para el cálculo de demandas térmicas. Los detalles del traspaso se muestran en la Error! Reference source not found.. Este tipo de trabajo tiene las mismas ventajas y desventajas que el caso anterior.

Tabla 4: Resumen de traspasos BIM-BPS, Fuente: Elaboración Propia, 2013.

\begin{tabular}{|c|c|c|c|c|}
\hline $\begin{array}{l}\text { Elementos y } \\
\text { propiedades } \\
\text { que contiene } \\
\text { el archivo } \\
\text { exportación }\end{array}$ & $\begin{array}{l}\text { gbXML: } \\
\text { Revit a } \\
\text { Ecotect }\end{array}$ & $\begin{array}{l}\text { PLUG-IN: } \\
\text { Revit a } \\
\text { Design } \\
\text { Builder. }\end{array}$ & $\begin{array}{l}\text { gbXML: } \\
\text { Revit a } \\
\text { Design } \\
\text { Builder }\end{array}$ & $\begin{array}{l}\text { RVT: } \\
\text { Revit a } \\
\text { Vasari }\end{array}$ \\
\hline Localización & $x$ & $x$ & $x$ & $x$ \\
\hline \multicolumn{5}{|l|}{ Geometría } \\
\hline Dimensiones & $x$ & $x$ & $x$ & $x$ \\
\hline Volumetría & $x$ & $x$ & $x$ & $x$ \\
\hline Zona & $x$ & $x$ & $x$ & \\
\hline $\begin{array}{l}\text { Uso de la } \\
\text { zona }\end{array}$ & $*$ & $*$ & $*$ & \\
\hline \multicolumn{5}{|l|}{$\begin{array}{l}\text { Tipo de } \\
\text { elemento }\end{array}$} \\
\hline Techo & $x^{1}$ & $x$ & $x$ & $x$ \\
\hline Cielo & $x^{1}$ & $x$ & $\mathrm{x}$ & $\mathrm{x}$ \\
\hline $\begin{array}{l}\text { Muro } \\
\text { exteriores }\end{array}$ & $x^{1}$ & $x$ & $x$ & $x$ \\
\hline $\begin{array}{l}\text { Muros } \\
\text { interiores }\end{array}$ & $x^{1}$ & $x^{3}$ & $x^{3}$ & $x$ \\
\hline Piso & $x^{1}$ & $\mathrm{x}$ & $x$ & $\mathrm{x}$ \\
\hline Puerta & $x^{2}$ & $\mathrm{x}$ & $x$ & $x$ \\
\hline Ventana & $x^{2}$ & $\mathrm{x}$ & $x$ & $\mathrm{x}$ \\
\hline \multicolumn{5}{|l|}{ Materialidad } \\
\hline Nombre & $*$ & $*$ & $*$ & \\
\hline Capas & $*$ & $*$ & $*$ & \\
\hline $\begin{array}{l}\text { Propiedades } \\
\text { Físicas }\end{array}$ & $*$ & $*$ & $*$ & \\
\hline \multicolumn{5}{|c|}{$\begin{array}{l}\text { * Información entregada por defecto } \\
{ }^{1} \text { para versiones Revit } 2013 \text { o superior, no se reconocen estos elementos. } \\
2 \text { en un } 80 \% \text { de los elementos son reconocidos, en caso contrario su } \\
\text { clasificación es de la "Guess" }\end{array}$} \\
\hline
\end{tabular}

A continuación se presenta un mapa (Figura 4) que resume los procesos de flujo de información entre BIM y BPS de acuerdo a una prestación específica presentados en este trabajo. En ella se pueden observar desde un punto de vista global los procesos de interoperabilidad.

\section{Conclusiones}

Se demuestra la existencia de estos tres ámbitos: BIM-BPSPrestaciones Energéticas y a su vez se demuestra el enorme potencial de interoperabilidad entre ellos. Se observa lo novedoso 
de la idea de mapear dichos procesos de intercambio de información y se abre un campo nuevo de para los arquitectos, investigadores, y consultores. En este mapeo se muestran los aspectos positivos y negativos de cada traspaso, en términos generales ahorra tiempos de modelado en el software BPS y solo hay que completar elementos de sombras y techumbres. Además de un aumento de la precisión y retroalimentación con BIM. EI mapeo es parcial y considera solo algunos software BIM-BPS, se espera en el futuro ampliarlo. Se espera con estos resultados estimular a los Consultores de Eficiencia Energética a usar modelos BIM y a su vez a los arquitectos a entregarlos con los requerimientos necesarios. Se hacen necesarios protocolos que definan los flujos de información (tales como la GSA en Estados Unidos). Se demuestra el potencial que Chile tiene para explotar el campo del BEM (Building Energy Modeling) por parte de los consultores, investigadores y académicos. Para avanzar en la completa fusión y sincronía de estos tres campos es necesario mejorar el nivel de conocimiento experto en las universidades incluyendo estas temáticas en los ramos de pre y postgrado. Finalmente los mas importante es alejar los prejuicios de los modeladores de Eficiencia Energética acerca de la eficiencia de los formatos DXF, gbXML, ifcXML que fue la motivación inicial de esta investigación.

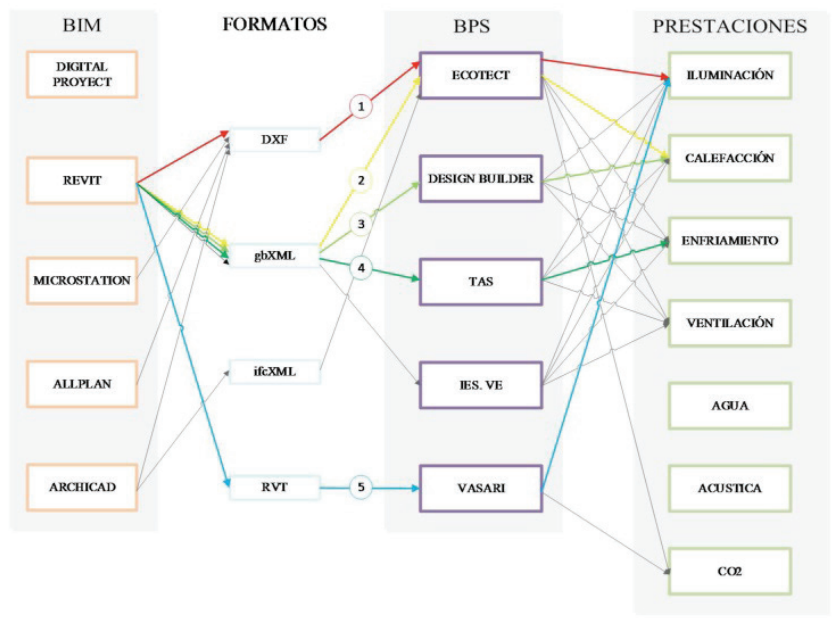

Figura 4: Mapa de flujo de información entre BIM y BPS según prestación específica. Fuente: elaboración propia, realizado en Microsoft Visio 2013.

\section{Agradecimientos}

Agradecemos a la Comisión Nacional de Ciencia y Tecnología (CONICYT) de Chile por incentivar la investigación en estas aéreas a través de su programa FONDECYT.

\section{Referencias}

AChEE (Agencia Chilena de Eficiencia Energética). (2012) Guía de eficiencia energética para establecimientos de Salud.
AChEE (Agencia Chilena de Eficiencia Energética). (2012) Guía de eficiencia energética para establecimientos educacionales.

Ali, S. (2010). Analysis Of Procedures And Workflow For Co nducting Energy Analysis Using Autodesk Revit, gbXML and Trace 700, SimBuild 2010, Fourth National Conference of IBPSA-USA, New York City, New York.

Attia, S., Beltran, L., Herde, A. de \& Hensen, JLM (2009)." Architect Friendly": a comparison of ten different building performance simulation tools.

Bazjanac, V. (2005). Model based cost and energy performance estimation during schematic design. In 22nd Conference on Information Technology in Construction (pp. 677-688).

Bazjanac, V. (2008). IFC BIM-based methodology for semi-automated building energy performance simulation. LBNL-919E.

Cho, C. S., Chen, D., \& Woo, S. (2011). Building Information Modeling (Bim)-Based Design Of Energy Efficient Buildings, Technical Report, S31-1.

Eastman, C., Teicholz, P., Sacks, R., \& Liston, K. (2011). BIM handbook: A guide to building information modeling for owners, managers, designers, engineers and contractors. Wiley. com.

Gonzales, A. (2012). Evaluación De Herramientas De Simulación Energética Para Viviendas En Concepción, MSc Thesis, Universidad del Bío-Bío, Chile

Graneros, A. (2013). Aprendizaje integrado de Arquitectura: Mediante vistas interiores graduadas, Tésis de Doctorado, Universidad del BioBio, Chile.

Kumar, S. (2008). Interoperability between building information models (BIM) and energy analysis programs. ProQuest.

Lobos, D. (2011), BIM Supported Building Envelopes and Space Layout based on a case study in South America, Bauhaus University Weimar Press, Weimar.

Ministerio de Obras Públicas. (2012). Manual de la Gestión Energética en Edificios Públicos. Santiago, Chile.

O’Donnell, J. T., Maile, T., Cody Rose, N., Mrazović, E. M., Regnier, C., Parrish, K., \& Bazjanac, V. (2013). Transforming BIM to BEM: Generation of Building Geometry for the NASA Ames Sustainability Base BIM.

Osello, A., Cangialosi, G., Dalmasso, D., Di Paolo, A., Turco, M. L., Piumatti, P., \& Vozzola, M. (2011) Architecture Data And Energy Efficiency Simulations: Bim And Interoperability Standards. Proceedings of Building Simulation 2011, Sydney.

Tse, T. C. (2009). The interoperability of building information models and document models in the Hong Kong construction industry.

U.S. Departament of Energy (http://apps1.eere.energy.gov/buildings/tools_directory)

U.S. General Services Administration Public Buildings Service. (2012). GSA Building Information Modeling Guide Series 05, Energy Performance and Operations.

WU, W. and ISSA, R.R.A., (2010). Feasibility of integrating building information modeling and LEED ${ }^{\circledR}$ certification process. In Computing in Civil and Building Engineering, Proceedings of the International Conference, W. TIZANI (Editor), 30 June-2 July, Nottingham, UK, Nottingham University Press, 81, 161, ISBN 978-1-907284-60-1 